\title{
HAZARD RATE ORDERING OF ORDER STATISTICS AND SYSTEMS
}

\author{
JORGE NAVARRO,${ }^{*}$ Universidad de Murcia \\ MOSHE SHAKED, ${ }^{* *}$ University of Arizona
}

\begin{abstract}
Let $\boldsymbol{X}=\left(X_{1}, X_{2}, \ldots, X_{n}\right)$ be an exchangeable random vector, and write $X_{(1: i)}=$ $\min \left\{X_{1}, X_{2}, \ldots, X_{i}\right\}, 1 \leq i \leq n$. In this paper we obtain conditions under which $X_{(1: i)}$ decreases in $i$ in the hazard rate order. A result involving more general (that is, not necessarily exchangeable) random vectors is also derived. These results are applied to obtain the limiting behaviour of the hazard rate function of the lifetimes of various coherent systems in reliability theory. The notions of the Samaniego signatures and the minimal signatures of such systems are extensively used in the paper. An interesting relationship between these two signatures is obtained. The results are illustrated in a series of examples.
\end{abstract}

Keywords: Exchangeable distribution; signature; coherent system; hazard rate order; stochastic order; Freund distribution; hazard gradient; cause specificity; Farlie-GumbelMorgenstern distribution

2000 Mathematics Subject Classification: Primary 60E15; 60K10

\section{Introduction}

Let $\boldsymbol{X}=\left(X_{1}, X_{2}, \ldots, X_{n}\right)$ be an exchangeable random vector, and write $X_{(1: i)}=\min \left\{X_{1}\right.$, $\left.X_{2}, \ldots, X_{i}\right\}, 1 \leq i \leq n$. Intuitively we expect $X_{(1: i)}$ to get smaller as $i$ gets larger. Indeed, it is not hard to verify that $X_{(1: i)}$ decreases in $i$ in the usual stochastic order (see Section 2 for more details). In this paper we study the (possible) monotonicity of $X_{(1: i)}$ in the hazard rate order (see Section 2 for the exact definition of this order). The hazard rate order is stronger than the usual stochastic order, and it turns out that it is not always true that $X_{(1: i)}$ decreases in $i$ in the hazard rate order; this is shown in Section 2. The purpose of this paper is to obtain conditions under which $X_{(1: i)}$ indeed decreases in $i$ in the hazard rate order. This is also done in Section 2, where we actually obtain a result involving more general (that is, not necessarily exchangeable) random vectors. Throughout the paper, the notions 'increasing' and 'decreasing' are used in the weak sense.

In Section 3 we apply the results of Section 2 to obtain the limiting behaviour of the hazard rate function of the lifetimes of various coherent systems in reliability theory. The notions of the Samaniego signatures and the minimal signatures of such systems are extensively used in

\footnotetext{
Received 29 April 2005; revision received 17 January 2006.

* Postal address: Facultad de Matematicas, Universidad de Murcia, 30100 Murcia, Spain.

Email address: jorgenav@um.es

** Postal address: Department of Mathematics, University of Arizona, Tucson, AZ 85721, USA.

Email address: shaked@math.arizona.edu

Partially supported by the Ministerio de Ciencia y Tecnologia under grant BFM2003-02947 and Fundacion Seneca under grant 00698/PI/04.
} 
that section. There we also obtain an interesting relationship between these two signatures. The results are illustrated in a series of examples.

\section{A basic result}

Let $\boldsymbol{X}=\left(X_{1}, X_{2}, \ldots, X_{n}\right)$ be a random vector with an absolutely continuous distribution function $F$. Denote its survival function by $\bar{F}$, i.e.

$$
\bar{F}(\boldsymbol{x})=\bar{F}\left(x_{1}, x_{2}, \ldots, x_{n}\right)=\mathrm{P}\left\{X_{1}>x_{1}, X_{2}>x_{2}, \ldots, X_{n}>x_{n}\right\},
$$

and let $R=-\log \bar{F}$ be the corresponding multivariate hazard function. For $i=1,2, \ldots, n$, define $r^{(i)}$ by

$$
r^{(i)}\left(x_{1}, x_{2}, \ldots, x_{n}\right)=\frac{\partial}{\partial x_{i}} R\left(x_{1}, x_{2}, \ldots, x_{n}\right) \quad \text { for } \boldsymbol{x} \in\{\boldsymbol{x}: \bar{F}(\boldsymbol{x})>0\} .
$$

The vector $\boldsymbol{r}=\left(r^{(1)}, r^{(2)}, \ldots, r^{(n)}\right)$ is called the hazard gradient of $\boldsymbol{X}$; see Johnson and Kotz (1975) and Marshall (1975). Note that $r^{(i)}(\boldsymbol{x})$ can be interpreted as the conditional hazard rate of $X_{i}$ evaluated at $x_{i}$, given that $X_{j}>x_{j}$ for all $j \neq i$; i.e.

$$
r^{(i)}(\boldsymbol{x})=\frac{f_{i}\left(x_{i} \mid X_{j}>x_{j}, j \neq i\right)}{\bar{F}_{i}\left(x_{i} \mid X_{j}>x_{j}, j \neq i\right)}
$$

where $f_{i}\left(\cdot \mid X_{j}>x_{j}, j \neq i\right)$ and $\bar{F}_{i}\left(\cdot \mid X_{j}>x_{j}, j \neq i\right)$ are the conditional density and survival functions of $X_{i}$, given that $X_{j}>x_{j}$ for all $j \neq i$. For convenience, we set $r^{(i)}(\boldsymbol{x})=\infty$ for all $x \in\{x: \bar{F}(x)=0\}$.

For any subset $P \subseteq\{1,2, \ldots, n\}$, define

$$
Y_{P}=\min _{i \in P} X_{i}
$$

Let

$$
1_{P}(i)=\left\{\begin{array}{ll}
0 & \text { if } i \notin P, \\
1 & \text { if } i \in P,
\end{array} \quad \mathbf{1}_{P}=\left(1_{P}(1), 1_{P}(2), \ldots, 1_{P}(n)\right), \quad \text { and } \quad \mathbf{1}_{P^{\mathrm{c}}}=\mathbf{1}-\mathbf{1}_{P},\right.
$$

where $\mathbf{1}=(1,1, \ldots, 1)$ and $P^{\mathrm{c}}$ denotes the complement of $P$ in $\{1,2, \ldots, n\}$. Also let

$$
\infty \cdot 1_{P^{\mathrm{c}}}(i)= \begin{cases}0 & \text { if } i \notin P^{\mathrm{c}} \\ \infty & \text { if } i \in P^{\mathrm{c}}\end{cases}
$$

and $\infty \cdot \mathbf{1}_{P^{\mathrm{c}}}=\left(\infty \cdot 1_{P^{\mathrm{c}}}(1), \infty \cdot 1_{P^{\mathrm{c}}}(2), \ldots, \infty \cdot 1_{P^{\mathrm{c}}}(n)\right)$. Then the survival function, $\bar{G}_{P}$, of $Y_{P}$ can be expressed as

$$
\bar{G}_{P}(t)=\bar{F}\left(t \cdot \mathbf{1}_{P}-\infty \cdot \mathbf{1}_{P^{\mathrm{c}}}\right), \quad t \in \mathbb{R} .
$$

Alternatively, $\bar{G}_{P}(t)=\bar{F}_{P}(t, t, \ldots, t)$, where $\bar{F}_{P}$ denotes the survival function of $\left\{X_{i}\right.$ : $i \in P\}$. Denoting by $\left(\partial / \partial x_{i}\right) \bar{F}$ the partial derivative of $\bar{F}$ with respect to its $i$ th argument, the density function, $g_{P}$, of $Y_{P}$ can be obtained by differentiating $\bar{G}_{P}$ as follows:

$$
g_{P}(t)=-\sum_{i \in P} \frac{\partial}{\partial x_{i}} \bar{F}\left(t \cdot \mathbf{1}_{P}-\infty \cdot \mathbf{1}_{P^{\mathrm{c}}}\right), \quad t \in \mathbb{R} .
$$


Therefore, the hazard rate function, $r_{P}$, of $Y_{P}$ is given by

$$
r_{P}(t)=\frac{g_{P}(t)}{\bar{G}_{P}(t)}=\sum_{i \in P} r^{(i)}\left(t \cdot \mathbf{1}_{P}-\infty \cdot \mathbf{1}_{P^{\mathrm{c}}}\right), \quad t \in \mathbb{R} .
$$

Theorem 2.1. Let $\left(X_{1}, X_{2}, \ldots, X_{n}\right)$ be a random vector with an absolutely continuous distribution function. Let $P$ and $Q$ be two subsets of $\{1,2, \ldots, n\}$ such that $P \subset Q$. If

$$
r^{(i)}\left(t \cdot \mathbf{1}_{P}-\infty \cdot \mathbf{1}_{P^{\mathrm{c}}}\right) \leq r^{(i)}\left(t \cdot \mathbf{1}_{Q}-\infty \cdot \mathbf{1}_{Q^{\mathrm{c}}}\right), \quad t \in \mathbb{R}, i \in P,
$$

then

$$
r_{P}(t) \leq r_{Q}(t), \quad t \in \mathbb{R} .
$$

Proof. For each $t \in \mathbb{R}$, we note that

$$
\begin{aligned}
r_{P}(t) & =\sum_{i \in P} r^{(i)}\left(t \cdot \mathbf{1}_{P}-\infty \cdot \mathbf{1}_{P^{\mathrm{c}}}\right) \\
& \leq \sum_{i \in P} r^{(i)}\left(t \cdot \mathbf{1}_{Q}-\infty \cdot \mathbf{1}_{Q^{\mathrm{c}}}\right) \\
& \leq \sum_{i \in Q} r^{(i)}\left(t \cdot \mathbf{1}_{Q}-\infty \cdot \mathbf{1}_{Q^{\mathrm{c}}}\right) \\
& =r_{Q}(t)
\end{aligned}
$$

where the first inequality follows from (2.2) and the second inequality follows from $P \subset Q$.

The conclusion, (2.3), of Theorem 2.1 can be rewritten as

$$
Y_{P} \geq_{\mathrm{hr}} Y_{Q}
$$

where ' $\leq \mathrm{hr}$ ' denotes the hazard rate stochastic order; see, e.g. Shaked and Shanthikumar (1994). Inequality (2.4) implies that

$$
Y_{P} \geq_{\text {st }} Y_{Q}
$$

where ' $\leq_{\text {st }}$ ' denotes the usual stochastic order; again see, e.g. Shaked and Shanthikumar (1994). Intuitively, (2.4) and (2.5) state that the smallest random variable from a set, $P$, of random variables is larger, in the respective stochastic order, than the smallest random variable from a larger set, $Q$, of random variables. Inequality (2.5) is weaker than inequality (2.4) because the hazard rate order implies the usual stochastic order. In fact, (2.5) always holds (i.e. a condition such as (2.2) is not needed) because $Y_{P} \geq Y_{Q}$ with certainty. However, in general, (2.4) need not always hold (i.e. without a condition such as (2.2)). This will be shown in Example 2.2 below.

In order to better understand the meaning of (2.2), let us look at it when $n=2$. Thus, let $\boldsymbol{X}=\left(X_{1}, X_{2}\right), P=\{1\}$, and $Q=\{1,2\}$. Then, on the one hand,

$$
r^{(1)}\left(t \cdot \mathbf{1}_{P}-\infty \cdot \mathbf{1}_{P^{\mathrm{c}}}\right)=r^{(1)}(t,-\infty)=\frac{f_{1}\left(t \mid X_{2}>-\infty\right)}{\bar{F}_{1}\left(t \mid X_{2}>-\infty\right)}=r_{1}(t),
$$

where $r_{1}$ is the marginal hazard rate function of $X_{1}$. On the other hand,

$$
r^{(1)}\left(t \cdot \mathbf{1}_{Q}-\infty \cdot \mathbf{1}_{Q^{c}}\right)=r^{(1)}(t, t)=\frac{f_{1}\left(t \mid X_{2}>t\right)}{\bar{F}_{1}\left(t \mid X_{2}>t\right)}=r_{1}\left(t \mid X_{2}>t\right),
$$


where $r_{1}\left(\cdot \mid X_{2}>t\right)$ denotes the hazard rate function of $\left[X_{1} \mid X_{2}>t\right]$. Thus, (2.2) reduces to

$$
r_{1}(t) \leq r_{1}\left(t \mid X_{2}>t\right), \quad t \in \mathbb{R} .
$$

Roughly speaking, (2.6) indicates a negative dependence relationship between $X_{1}$ and $X_{2}$ in the following sense. For ease of exposition, let us here assume that $X_{1}$ and $X_{2}$ are the respective positive lifetimes of two components, $A$ and $B$, say. Then, for $t>0,(2.6)$ means that the unconditional hazard rate of $X_{1}$ (i.e. conditioned on $X_{2}>0$ ) at time $t$ is smaller than the hazard rate of $X_{1}$, given that $X_{2}>t$, at time $t$. In other words, given that $X_{2}>t$, component $A$ is more likely to fail at time $t$ than it is when nothing is known about $X_{2}$ (except, of course, that it is positive).

Let us denote the right-hand side of (2.6) by $h_{1}$, and write it as

$$
h_{1}(t)=\lim _{\Delta t \downarrow 0} \frac{1}{\Delta t} \mathrm{P}\left\{t<X_{1} \leq t+\Delta t \mid X_{1}>t, X_{2}>t\right\}, \quad t \in \mathbb{R} .
$$

Similarly define $h_{2}$. In the biometrics literature the functions $h_{i}$ are called the cause-specific hazard rate functions; see, e.g. Holt (1978), Prentice et al. (1978), and references therein. In the setting of reliability theory the functions $h_{i}$ are called the initial hazard rate functions; see, e.g. Scarsini and Shaked (1999) and references therein. Thus, in the bivariate case, condition (2.6) means that the marginal hazard rate function of $X_{1}$ is pointwise no larger than the initial hazard rate function of $X_{1}$.

Condition (2.2) is sufficient, but not necessary, for the conclusion (2.3) to hold. This is shown in the following example.

Example 2.1. Let $\left(X_{1}, X_{2}\right)$ have the bivariate Freund (1961) distribution with a density function, $f$, given by

$$
f\left(x_{1}, x_{2}\right)= \begin{cases}\alpha \beta^{\prime} \mathrm{e}^{-\beta^{\prime} x_{2}-\left(\alpha+\beta-\beta^{\prime}\right) x_{1}} & \text { for } 0 \leq x_{1} \leq x_{2}, \\ \beta \alpha^{\prime} \mathrm{e}^{-\alpha^{\prime} x_{1}-\left(\alpha+\beta-\alpha^{\prime}\right) x_{2}} & \text { for } 0 \leq x_{2} \leq x_{1},\end{cases}
$$

where $\alpha, \beta, \alpha^{\prime}$, and $\beta^{\prime}$ are positive parameters. It is helpful to think of $X_{1}$ and $X_{2}$ as being the respective lifetimes of two components, $A$ and $B$, say. Then $\alpha$ is the failure rate of component $A$ when component $B$ is still functioning, and $\beta$ is the failure rate of component $B$ when component $A$ is still functioning. Furthermore, $\alpha^{\prime}$ is the failure rate of component $A$ after component $B$ has failed, and $\beta^{\prime}$ is the failure rate of component $B$ after component $A$ has failed. Take $P=\{1\}$ and $Q=\{1,2\}$. Then, obviously, $r_{Q}(t)=\alpha+\beta$ for $t>0$. The marginal density, $f_{1}$, of $X_{1}$ is given by (see Freund (1961))

$$
f_{1}\left(x_{1}\right)=\frac{1}{\alpha+\beta-\alpha^{\prime}}\left[\left(\alpha-\alpha^{\prime}\right)(\alpha+\beta) \mathrm{e}^{-(\alpha+\beta) x_{1}}+\alpha^{\prime} \beta \mathrm{e}^{-\alpha^{\prime} x_{1}}\right], \quad x_{1}>0,
$$

provided that $\alpha+\beta-\alpha^{\prime} \neq 0$. Thus, the survival function, $\bar{F}_{1}$, of $X_{1}$ is given by

$$
\bar{F}_{1}\left(x_{1}\right)=\frac{1}{\alpha+\beta-\alpha^{\prime}}\left[\left(\alpha-\alpha^{\prime}\right) \mathrm{e}^{-(\alpha+\beta) x_{1}}+\beta \mathrm{e}^{-\alpha^{\prime} x_{1}}\right], \quad x_{1}>0,
$$

and the hazard rate function of $X_{1}$, namely $r_{P}$, is given by

$$
r_{P}(t)=\frac{\left(\alpha-\alpha^{\prime}\right)(\alpha+\beta) \mathrm{e}^{-(\alpha+\beta) t}+\alpha^{\prime} \beta \mathrm{e}^{-\alpha^{\prime} t}}{\left(\alpha-\alpha^{\prime}\right) \mathrm{e}^{-(\alpha+\beta) t}+\beta \mathrm{e}^{-\alpha^{\prime} t}} \text { for } t>0 .
$$


It can be verified that $r_{P}(t) \leq r_{Q}(t)$ for all $t>0$, i.e. that (2.3) holds. (To verify that $r_{P}(t) \leq r_{Q}(t)$, we must consider two cases: (i) $\alpha+\beta-\alpha^{\prime}>0$, in which case both the numerator and the denominator of (2.7) are positive, and (ii) $\alpha+\beta-\alpha^{\prime}<0$, in which case both the numerator and the denominator of (2.7) are negative.)

However, if $\alpha<\alpha^{\prime}$ then (2.2) does not hold. With $P$ and $Q$ as above we have $r^{(1)}\left(t \cdot \mathbf{1}_{P}-\infty \cdot \mathbf{1}_{P^{\mathrm{c}}}\right)=r_{P}(t)$, whereas $r^{(1)}\left(t \cdot \mathbf{1}_{Q}-\infty \cdot \mathbf{1}_{Q^{\mathrm{c}}}\right)=r_{1}\left(t \mid X_{2}>t\right)=\alpha$. It is straightforward to see that $r_{P}(t)>\alpha$ for all $t>0$ (again, for the purpose of verifying this, note that if $\alpha+\beta-\alpha^{\prime}>0$ then both the numerator and the denominator of (2.7) are positive, and if $\alpha+\beta-\alpha^{\prime}<0$ then both the numerator and the denominator of (2.7) are negative). Thus, (2.2) fails to hold.

In the next example we show that (2.4), or, equivalently, (2.3), need not always hold (i.e. without a condition such as (2.2)).

Example 2.2. Let $\left(X_{1}, X_{2}\right)$ be a nonnegative random vector with the density function described in Figure 1. A straightforward computation shows that the marginal density, $f_{1}$, of $X_{1}$ is given by

$$
f_{1}\left(x_{1}\right)= \begin{cases}\left(2 x_{1}+1\right) \mathrm{e}^{-2 x_{1}}, & 0 \leq x_{1}<1, \\ \frac{35}{4} \mathrm{e}^{-\left(10 x_{1}-8\right)}+\frac{9}{4} \mathrm{e}^{-2 x_{1}}, & x_{1} \geq 1,\end{cases}
$$

and the survival function, $\bar{F}_{1}$, of $X_{1}$ is given by

$$
\bar{F}_{1}\left(x_{1}\right)= \begin{cases}\left(x_{1}+1\right) \mathrm{e}^{-2 x_{1}}, & 0 \leq x_{1}<1, \\ \frac{7}{8} \mathrm{e}^{-\left(10 x_{1}-8\right)}+\frac{9}{8} \mathrm{e}^{-2 x_{1}}, & x_{1} \geq 1 .\end{cases}
$$

Thus, the hazard rate function, $r_{1}$, of $X_{1}$ is given by

$$
r_{1}(t)= \begin{cases}\frac{2 t+1}{t+1}, & 0 \leq t<1, \\ \frac{70 \mathrm{e}^{-(10 t-8)}+18 \mathrm{e}^{-2 t}}{7 \mathrm{e}^{-(10 t-8)}+9 \mathrm{e}^{-2 t}}, & t \geq 1 .\end{cases}
$$

A lengthy yet again straightforward computation then shows that the hazard rate function, $r_{\min \left\{X_{1}, X_{2}\right\}}$, of $\min \left\{X_{1}, X_{2}\right\}$ is given by

$$
r_{\min \left\{X_{1}, X_{2}\right\}}(t)=2, \quad t \geq 0 .
$$

Let $P=\{1\}$ and $Q=\{1,2\}$. Then $r_{P}=r_{1}$ and $r_{Q}=r_{\min \left\{X_{1}, X_{2}\right\}}$. Since

$$
r_{P}(2)=\frac{70 \mathrm{e}^{-12}+18 \mathrm{e}^{-4}}{7 \mathrm{e}^{-12}+9 \mathrm{e}^{-4}}>2=r_{Q}(2),
$$

we see that (2.4) fails to hold.

A sufficient condition for (2.2) to hold is that

$$
r^{(i)}\left(x_{1}, x_{2}, \ldots, x_{n}\right) \text { is increasing in } x_{j}, \quad j \neq i, i=1,2, \ldots, n .
$$




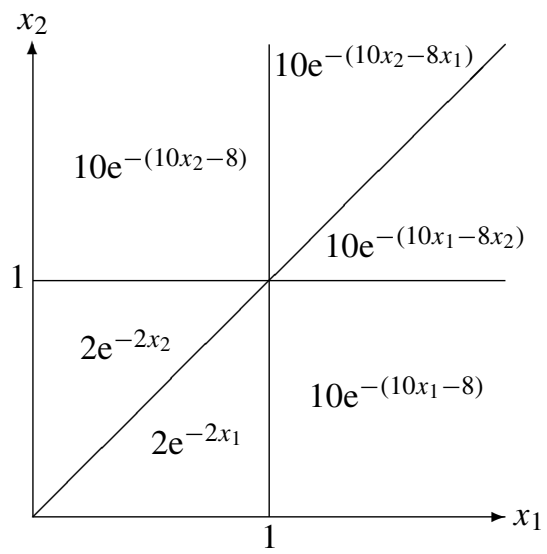

FIGURE 1: Density for Example 2.2.

This is easily seen to be equivalent to the requirement that

$$
\begin{array}{r}
\bar{F}\left(x_{1}, \ldots, x_{i-1}, x_{i}^{\prime}, x_{i+1}, \ldots, x_{n}\right) \\
\bar{F}\left(x_{1}, \ldots, x_{i-1}, x_{i}, x_{i+1}, \ldots, x_{n}\right) \\
\text { is decreasing in } x_{j}, j \neq i, \text { whenever } x_{i} \leq x_{i}^{\prime}, \\
i=1,2, \ldots, n .
\end{array}
$$

Condition (2.8) means that $\bar{F}$ is $\mathrm{RR}_{2}$ (reverse regular of order 2) in pairs; this is a notion of negative dependence that was studied in Block et al. (1982). They showed that some multivariate normal distributions, as well as the Dirichlet distribution, are $\mathrm{RR}_{2}$ in pairs; see also Karlin and Rinott (1980). Theorem 2.1 thus applies to these distributions.

If $X_{1}, X_{2}, \ldots, X_{n}$ are independent (but not necessarily identically distributed), absolutely continuous random variables, then (2.8) holds trivially. Recall that $X_{(1: i)}=\min \left\{X_{1}, X_{2}\right.$, $\left.\ldots, X_{i}\right\}, i=1,2, \ldots, n$. From Theorem 2.1 we thus find that if $X_{1}, X_{2}, \ldots, X_{n}$ are independent, then $X_{(1: 1)} \geq_{\mathrm{hr}} X_{(1: 2)} \geq_{\mathrm{hr}} \cdots \geq_{\mathrm{hr}} X_{(1: n)}$. This result can also be obtained from results of Korwar (2003).

In the following corollary we specialize Theorem 2.1 to the case in which $\left(X_{1}, X_{2}, \ldots, X_{n}\right)$ has an exchangeable distribution function. The corresponding multivariate hazard function, $R$, is then permutation symmetric. Therefore, each $r^{(i)}$ can be expressed by means of $r^{(1)}$ as follows:

$$
\begin{aligned}
& r^{(i)}\left(x_{1}, x_{2}, \ldots, x_{i-1}, x_{i}, x_{i+1}, \ldots, x_{n}\right) \\
& \quad=r^{(1)}\left(x_{i}, x_{2}, \ldots, x_{i-1}, x_{1}, x_{i+1}, \ldots, x_{n}\right), \quad i=2,3, \ldots, n .
\end{aligned}
$$

Corollary 2.1. Let $\left(X_{1}, X_{2}, \ldots, X_{n}\right)$ be a random vector with an absolutely continuous, exchangeable distribution function. If

$$
\begin{aligned}
& r^{(1)}(\underbrace{t, t, \ldots, t}_{i \text { times }}, \underbrace{-\infty,-\infty, \ldots,-\infty}_{n-i \text { times }}) \\
& \quad \leq r^{(1)}(\underbrace{t, t, \ldots, t}_{i+1 \text { times }}, \underbrace{-\infty,-\infty, \ldots,-\infty}_{n-i-1 \text { times }}), \quad t \in \mathbb{R}, i=1,2, \ldots, n-1,
\end{aligned}
$$

then

$$
X_{(1: 1)} \geq_{\mathrm{hr}} X_{(1: 2)} \geq_{\mathrm{hr}} \cdots \geq_{\mathrm{hr}} X_{(1: n)}
$$


If (2.9) is not imposed then (2.10) need not be true; this follows from a variant of Example 2.2 in which $\left(X_{1}, X_{2}\right)$ has an exchangeable distribution function.

Note that

$$
r^{(1)}(\underbrace{t, t, \ldots, t}_{i \text { times }}, \underbrace{-\infty,-\infty, \ldots,-\infty}_{n-i \text { times }})=r_{1 \mid 23 \cdots i}\left(t \mid X_{2}>t, X_{3}>t, \ldots, X_{i}>t\right),
$$

where $r_{1 \mid 23 \ldots i}\left(\cdot \mid X_{2}>t, X_{3}>t, \ldots, X_{i}>t\right)$ denotes the hazard rate function of $X_{1}$ conditioned on the given condition. Thus, (2.9) implies that

$$
\begin{aligned}
r_{1}(t) \leq r_{1 \mid 2}\left(t \mid X_{2}>t\right) & \leq r_{1 \mid 23}\left(t \mid X_{2}>t, X_{3}>t\right) \\
& \leq \cdots \leq r_{1 \mid 23 \cdots n}\left(t \mid X_{2}>t, X_{3}>t, \ldots, X_{n}>t\right), \quad t \in \mathbb{R},
\end{aligned}
$$

where $r_{1}$ is the marginal hazard rate function of $X_{1}$. Again, this is a condition that indicates a negative dependence relationship among the $X_{i}$. For example, if the $X_{i}$ are the lifetimes of $n$ components, then the above sequence of inequalities roughly says that the larger is the number of known 'old' components (i.e. older than $t$ ), the more likely is it that any other component will not become 'much older' (i.e. fail shortly after time $t$ ).

\section{Tail behaviour of system hazard rate functions}

In this section we describe some applications of Theorem 2.1 and Corollary 2.1 in reliability theory. Let $X_{1}, X_{2}, \ldots, X_{n}$ be the lifetimes of $n$ components of a coherent system; a comprehensive study of coherent systems can be found in Barlow and Proschan (1975). Let $\tau$ be the life function of the coherent system; i.e. $T=\tau\left(X_{1}, X_{2}, \ldots, X_{n}\right)$ is the lifetime of the system with the above components. Then $T$ can be represented as

$$
T=\max _{1 \leq j \leq s} \min _{i \in P_{j}} X_{i},
$$

where the sets $P_{1}, P_{2}, \ldots, P_{s}$ are called the minimal path sets of the coherent system.

\subsection{Results based on minimal signatures and path sets}

Consider the case in which $X_{1}, X_{2}, \ldots, X_{n}$ have an exchangeable distribution function. As in Section 2 we write $X_{(1: i)}=\min \left\{X_{1}, X_{2}, \ldots, X_{i}\right\}$, and we let $\bar{F}_{(1: i)}$ denote the survival function of $X_{(1: i)}, i=1,2, \ldots, n$. Under the above assumption of exchangeability, it has been noted (see Navarro et al. (2004)) that the survival function, $\bar{F}$, of $T$ can be expressed as a linear combination of the survival functions, $\bar{F}_{(1, i)}$, of $X_{(1: i)}, i=1,2, \ldots, n$, i.e. that

$$
\bar{F}=\sum_{i=1}^{n} a_{i} \bar{F}_{(1: i)}
$$

for some negative and nonnegative integers $a_{i}$ that obviously satisfy $\sum_{i=1}^{n} a_{i}=1$. Navarro et al. (2004) called the vector $\left(a_{1}, a_{2}, \ldots, a_{n}\right)$ the minimal signature of the coherent system. The minimal signature of a coherent system can be obtained also from Equation (3.1) of Block et al. (2003). The minimal signature of a series system of two components is of course $\left(a_{1}, a_{2}\right)=$ $(1,0)$, and the minimal signature of a parallel system of two components is $\left(a_{1}, a_{2}\right)=(2,-1)$. The minimal signatures of all five coherent systems of size 3 , and of all twenty coherent systems of size 4 are given in Tables 1 and 2 . In these tables, and later in the paper, we denote by $X_{(i: n)}$ the $i$ th order statistic among $X_{1}, X_{2}, \ldots, X_{n}$. 
TABLE 1: Minimal signatures of all systems of size 3.

\begin{tabular}{lcc}
\hline \multicolumn{1}{c}{ System } & $\tau\left(X_{1}, X_{2}, X_{3}\right)$ & $\left(a_{1}, a_{2}, a_{3}\right)$ \\
\hline Series & $X_{(1: 3)}$ & $(0,0,1)$ \\
Series-parallel & $\min \left\{X_{1}, \max \left\{X_{2}, X_{3}\right\}\right\}$ & $(0,2,-1)$ \\
2-out-of-3 & $X_{(2: 3)}$ & $(0,3,-2)$ \\
Parallel-series & $\max \left\{X_{1}, \min \left\{X_{2}, X_{3}\right\}\right\}$ & $(1,1,-1)$ \\
Parallel & $X_{(3: 3)}$ & $(3,-3,1)$ \\
\hline
\end{tabular}

TABLE 2: Minimal signatures of all systems of size 4.

\begin{tabular}{ccc}
\hline System & $\tau\left(X_{1}, X_{2}, X_{3}, X_{4}\right)$ & $\left(a_{1}, a_{2}, a_{3}, a_{4}\right)$ \\
\hline 1 & $X_{(1: 4)}$ & $(0,0,0,1)$ \\
2 & $\max \left\{\min \left\{X_{1}, X_{2}, X_{3}\right\}, \min \left\{X_{2}, X_{3}, X_{4}\right\}\right\}$ & $(0,0,2,-1)$ \\
3 & $\left.\min \left\{X_{1}, \max 2 \leq i<j \leq 4\right) \min \left\{X_{i}, X_{j}\right\}\right\}$ & $(0,0,3,-2)$ \\
4 & $\min \left\{X_{1}, \max \left\{X_{2}, X_{3}\right\}, \max \left\{X_{2}, X_{4}\right\}\right\}$ & $(0,1,1,-1)$ \\
5 & $\min \left\{X_{1}, \max \left\{X_{2}, X_{3}, X_{4}\right\}\right\}$ & $(0,3,-3,1)$ \\
6 & $X_{(2: 4)}$ & $(0,0,4,-3)$ \\
7 & $\max \left\{\min \left\{X_{1}, X_{2}\right\}, \min \left\{X_{1}, X_{3}, X_{4}\right\}, \min \left\{X_{2}, X_{3}, X_{4}\right\}\right\}$ & $(0,1,2,-2)$ \\
8 & $\max \left\{\min \left\{X_{1}, X_{2}\right\}, \min \left\{X_{3}, X_{4}\right\}\right\}$ & $(0,2,0,-1)$ \\
9 & $\max \left\{\min \left\{X_{1}, X_{2}\right\}, \min \left\{X_{1}, X_{3}\right\}, \min \left\{X_{2}, X_{3}, X_{4}\right\}\right\}$ & $(0,2,0,-1)$ \\
10 & $\max \left\{\min \left\{X_{1}, X_{2}\right\}, \min \left\{X_{2}, X_{3}\right\}, \min \left\{X_{3}, X_{4}\right\}\right\}$ & $(0,3,-2,0)$ \\
11 & $\max \left\{\min \left\{X_{1}, \max \left\{X_{2}, X_{3}, X_{4}\right\}\right\}, \min \left\{X_{2}, X_{3}, X_{4}\right\}\right\}$ & $(0,3,-2,0)$ \\
12 & $\max \left\{\min \left\{X_{1}, \max \left\{X_{2}, X_{3}, X_{4}\right\}\right\}, \min \left\{X_{2}, X_{3}\right\}\right\}$ & $(0,4,-4,1)$ \\
13 & $\min \left\{\max \left\{X_{1}, X_{2}\right\}, \max \left\{X_{3}, X_{4}\right\}\right\}$ & $(0,4,-4,1)$ \\
14 & $\min \left\{\max \left\{X_{1}, X_{2}\right\}, \max \left\{X_{1}, X_{3}, X_{4}\right\}, \max \left\{X_{2}, X_{3}, X_{4}\right\}\right\}$ & $(0,5,-6,2)$ \\
15 & $X_{(3: 4)}$ & $(0,6,-8,3)$ \\
16 & $\max \left\{X_{1}, \min \left\{X_{2}, X_{3}, X_{4}\right\}\right\}$ & $(1,0,1,-1)$ \\
17 & $\max \left\{X_{1}, \min \left\{X_{2}, X_{4}\right\}, \min \left\{X_{3}, X_{4}\right\}\right\}$ & $(1,2,-3,1)$ \\
18 & $\max \left\{X_{1}, \max x_{2 \leq i<j \leq 4} \min \left\{X_{i}, X_{j}\right\}\right\}$ & $(1,3,-5,2)$ \\
19 & $\max \left\{X_{1}, X_{2}, \min \left\{X_{3}, X_{4}\right\}\right\}$ & $(2,0,-2,1)$ \\
20 & $X_{(4: 4)}$ & $(4,-6,4,-1)$ \\
\hline
\end{tabular}

There exist several results in the literature which show, under certain conditions, that the asymptotic behaviour, as $t \rightarrow \infty$, of the failure rate function of a mixture is the same as the asymptotic behaviour of the failure rate of the strongest member of the mixture; see Block and Joe (1997), Block et al. (2003), Navarro and Hernandez (2004a), and Li (2005). Thus, we might expect similar results to hold for $\bar{F}$ in (3.1), although that $\bar{F}$ is not a mixture of the $\bar{F}_{(1: i)}$, since some of the $a_{i}$ are negative.

In order to develop this idea let us now consider a general linear combination of survival functions (called a 'generalized mixture' in Baggs and Nagaraja (1996) and in Navarro and Hernandez (2004b)) of the form

$$
\bar{G}=\sum_{i=1}^{n} w_{i} \bar{G}_{i},
$$

where the $\bar{G}_{i}$ are survival functions and the $w_{i}$ are some nonzero (and not necessarily positive) constants that satisfy $\sum_{i=1}^{n} w_{i}=1$. In general, $\bar{G}$ in (3.2) need not be a survival 
function - some conditions on the $w_{i}$ are needed in order to guarantee that it is (see Navarro and Hernandez (2004b)). However, for our purposes we can assume here that these conditions are satisfied and that $\bar{G}$ in (3.2) is a survival function. We also suppose below that the $\bar{G}_{i}$ are absolutely continuous with respective hazard rate functions $r_{1}, r_{2}, \ldots, r_{n}$. Then $\bar{G}$ is also absolutely continuous, and we denote its hazard rate function by $r$. The following lemmas will be used in the sequel; they are special cases of results in Navarro and Hernandez (2004b).

Lemma 3.1. Let $\bar{G}_{1}$ and $\bar{G}_{2}$ be two absolutely continuous survival functions with respective hazard rate functions $r_{1}$ and $r_{2}$. If

$$
\liminf _{t \rightarrow \infty} \frac{r_{2}(t)}{r_{1}(t)}>1
$$

then

$$
\lim _{t \rightarrow \infty} \frac{\bar{G}_{2}(t)}{\bar{G}_{1}(t)}=0 .
$$

Lemma 3.2. Let $\bar{G}$ be as in (3.2). If

$$
\liminf _{t \rightarrow \infty} \frac{r_{i}(t)}{r_{1}(t)}>1, \quad i=2,3, \ldots, n,
$$

then $w_{1}>0$.

Lemma 3.3. Let $\bar{G}$ be as in (3.2). If (3.3) holds and

$$
\limsup _{t \rightarrow \infty} \frac{r_{i}(t)}{r_{1}(t)}<\infty, \quad i=2,3, \ldots, n,
$$

then

$$
\lim _{t \rightarrow \infty} \frac{r(t)}{r_{1}(t)}=1 .
$$

A variation of Lemma 3.3 that will be used in the sequel is the following; it also follows from results of Navarro and Hernandez (2004b).

Lemma 3.4. Let $\bar{G}$ be as in (3.2). If (3.3) holds and

$$
\lim _{t \rightarrow \infty} \frac{r_{i}(t) \bar{G}_{i}(t)}{r_{1}(t) \bar{G}_{1}(t)}=0, \quad i=2,3, \ldots, n,
$$

then

$$
\lim _{t \rightarrow \infty} \frac{r(t)}{r_{1}(t)}=1 .
$$

Note that the limit in (3.5) can be written as $\lim _{t \rightarrow \infty}\left(g_{i}(t) / g_{1}(t)\right)=0$, where $g_{i}$ and $g_{1}$ are respectively the density functions corresponding to $\bar{G}_{i}$ and $\bar{G}_{1}$. If (3.3) holds then the conclusion of Lemma 3.4 holds even under the weaker condition that $\lim _{t \rightarrow \infty}\left(g_{i}(t) / g_{1}(t)\right)$ exists and is finite, for $i=2,3, \ldots, n$; this follows from Lemma 3.1 and l'Hôpital's rule (i.e. $\left.\lim _{t \rightarrow \infty}\left(\bar{G}_{i}(t) / \bar{G}_{1}(t)\right)=\lim _{t \rightarrow \infty}\left(g_{i}(t) / g_{1}(t)\right)\right)$.

Note that if (3.3) holds then (3.4) implies (3.5). Thus, formally, Lemma 3.4 is a stronger result than is Lemma 3.3. In fact, the implication just mentioned is strict; this will be seen in the sequel (see Example 3.1). Here we have given both lemmas because both are used below. 
We can now apply Corollary 2.1 to state and prove a result which shows that for many a coherent system the asymptotic behaviour of the failure rate function of its lifetime is the same as the asymptotic behaviour of the failure rate of a certain series system. For this purpose we consider an exchangeable random vector $\left(X_{1}, X_{2}, \ldots, X_{n}\right)$, and we respectively denote by $\bar{F}_{(1: i)}$ and $r_{(1: i)}$ the survival function and the hazard rate function of $X_{(1: i)}, i=1,2, \ldots, n$.

Theorem 3.1. Let $\left(X_{1}, X_{2}, \ldots, X_{n}\right)$ be an absolutely continuous, exchangeable random vector satisfying (2.9). Let $T=\tau\left(X_{1}, X_{2}, \ldots, X_{n}\right)$, where $\tau$ is a coherent life function. Suppose that the minimal signature of $\tau$ is of the form $\left(0,0, \ldots, 0, a_{i}, a_{i+1}, \ldots, a_{m}, 0,0\right.$, $\ldots, 0)$ for $1 \leq i<m \leq n$, with $a_{i} \neq 0$ and $a_{m} \neq 0$. If

$$
\limsup _{t \rightarrow \infty} \frac{r_{(1: m)}(t)}{r_{(1: i)}(t)}<\infty
$$

then $a_{i}>0$ and the hazard rate function, $r$, of $T$ satisfies

$$
\lim _{t \rightarrow \infty} \frac{r(t)}{r_{(1: i)}(t)}=1 \text {. }
$$

Proof. In the exchangeable case, (2.1) yields

$$
r_{(1: j)}(t)=j \phi_{j}(t), \quad t \in \mathbb{R},
$$

for $j=1,2, \ldots, n$, where

$$
\phi_{j}(t)=r^{(1)}(\underbrace{t, t, \ldots, t}_{j \text { times }}, \underbrace{-\infty,-\infty, \ldots,-\infty}_{n-j \text { times }}) .
$$

Hence, for $j=i+1, i+2, \ldots, m$, we have

$$
\liminf _{t \rightarrow \infty} \frac{r_{(1: j)}(t)}{r_{(1: i)}(t)} \geq \liminf _{t \rightarrow \infty} \frac{r_{(1: i+1)}(t)}{r_{(1: i)}(t)}=\frac{i+1}{i} \liminf _{t \rightarrow \infty} \frac{\phi_{i+1}(t)}{\phi_{i}(t)} \geq \frac{i+1}{i}>1,
$$

where the first inequality follows from (2.10) and the second inequality follows from (2.9). Furthermore, from (2.10) and (3.6) we see that

$$
\limsup _{t \rightarrow \infty} \frac{r_{(1: j)}(t)}{r_{(1: i)}(t)}<\infty, \quad j=i+1, i+2, \ldots, m .
$$

Since the survival function, $\bar{F}$, of $T$ satisfies (3.1), it follows from Lemma 3.2 that $a_{i}>0$, and it follows from Lemma 3.3 that $\lim _{t \rightarrow \infty}\left(r(t) / r_{(1: i)}(t)\right)=1$.

Note that (3.6) can also be written as $\lim _{\sup } \operatorname{si\infty }_{t \rightarrow}\left(\phi_{m}(t) / \phi_{i}(t)\right)<\infty$.

In Example 3.2 below it is shown that for every choice of $i, m$, and $n$ with $1 \leq i<m \leq n$, there exists an absolutely continuous, exchangeable random vector $\left(X_{1}, X_{2}, \ldots, X_{n}\right)$ such that (2.9) and (3.6) hold. Thus, every coherent system has a minimal signature of the form $\left(0,0, \ldots, 0, a_{i}, a_{i+1}, \ldots, a_{m}, 0,0, \ldots, 0\right)$, with $a_{i}>0$ and $a_{m} \neq 0$. Common examples are the $k$-out-of- $n$ systems; for such systems the minimal signature is of the form above with $i=k$ and $a_{k}=\left(\begin{array}{l}n \\ k\end{array}\right)$. The lifetime of a $k$-out-of- $n$ system with component lifetimes $X_{1}, X_{2}, \ldots, X_{n}$ is $X_{(n+k-1: n)}$. Thus, from Theorem 3.1 we obtain the following corollary. 
Corollary 3.1. Let $\left(X_{1}, X_{2}, \ldots, X_{n}\right)$ be an absolutely continuous, exchangeable random vector satisfying (2.9). Let $1 \leq k \leq n$. If

$$
\limsup _{t \rightarrow \infty} \frac{r_{(1: n)}(t)}{r_{(1: k)}(t)}<\infty
$$

then

$$
\lim _{t \rightarrow \infty} \frac{r_{(n-k+1: n)}(t)}{r_{(1: k)}(t)}=1 .
$$

Below we will see a typical example in which (2.9) and (3.8) hold, and in which the limit $\lim _{t \rightarrow \infty} r_{(1: k)}$ is both finite and easy to compute (see Example 3.2, which includes as a special case that in which $X_{1}, X_{2}, \ldots, X_{n}$ are independent and identically distributed exponential random variables). Then, from Corollary 3.1 we will be able to obtain $\lim _{t \rightarrow \infty} r_{(n-k+1: n)}(t)$ explicitly (i.e. $\lim _{t \rightarrow \infty} r_{(n-k+1: n)}(t)$ will be equal to $\left.\lim _{t \rightarrow \infty} r_{(1: k)}(t)\right)$.

A variation of Theorem 3.1 is the following.

Theorem 3.2. Let $\left(X_{1}, X_{2}, \ldots, X_{n}\right)$ be an absolutely continuous, exchangeable random vector satisfying (2.9). Let $T=\tau\left(X_{1}, X_{2}, \ldots, X_{n}\right)$, where $\tau$ is a coherent life function. Suppose that the minimal signature of $\tau$ is of the form $\left(0,0, \ldots, 0, a_{i}, a_{i+1}, \ldots, a_{m}, 0,0\right.$, $\ldots$,0) for $1 \leq i<m \leq n$, with $a_{i} \neq 0$ and $a_{m} \neq 0$. If

$$
\lim _{t \rightarrow \infty} \frac{r_{(1: j)}(t) \bar{F}_{(1: j)}(t)}{r_{(1: i)}(t) \bar{F}_{(1: i)}(t)}=0, \quad j=i+1, \ldots, m,
$$

then $a_{i}>0$ and the hazard rate function, $r$, of $T$ satisfies

$$
\lim _{t \rightarrow \infty} \frac{r(t)}{r_{(1: i)}(t)}=1 \text {. }
$$

Proof. From the proof of Theorem 3.1 it follows that (3.7) holds. Thus, by Lemma 3.4, the inequalities (3.7) and (3.9) imply the stated result.

Note that the limit in (3.9) can be written as $\lim _{t \rightarrow \infty}\left(f_{(1: j)}(t) / f_{(1: i)}(t)\right)=0$, where $f_{(1: j)}$ and $f_{(1: i)}$ are respectively the density functions of $X_{(1: j)}$ and $X_{(1: i)}$.

Also note that if (2.9) holds then (3.6) implies (3.9). Thus, Theorem 3.2 is a stronger result than is Theorem 3.1. In fact, the implication just mentioned is strict; this will be seen in Example 3.1. Here we state both Theorems 3.1 and 3.2 because sometimes it is easier to verify the stronger condition, (3.6), than it is to verify (3.9).

We now give a few examples in which the conditions of Theorems 3.1 and 3.2 are satisfied, and in which the conclusions of these theorems can therefore be applied.

Example 3.1. Let $\left(X_{1}, X_{2}\right)$ have the Gumbel exponential distribution with survival function given by

$$
\bar{F}\left(x_{1}, x_{2}\right)=\exp \left\{-x_{1}-x_{2}-\theta x_{1} x_{2}\right\}, \quad x_{1} \geq 0, x_{2} \geq 0,
$$

where $0 \leq \theta \leq 1$. Here, for $t \geq 0$, we have $r^{(1)}(t, 0)=1 \leq 1+\theta t=r^{(1)}(t, t)$, so (2.9) holds. Also,

$$
r_{(1: 1)}(t)=1 \quad \text { and } \quad r_{(1: 2)}(t)=2+2 \theta t, \quad t \geq 0 .
$$

Hence,

$$
\frac{r_{(1: 2)}(t) \bar{F}_{(1: 2)}(t)}{r_{(1: 1)}(t) \bar{F}_{(1: 1)}(t)}=(2+2 \theta t) \mathrm{e}^{-t-\theta t^{2}} \rightarrow 0 \quad \text { as } t \rightarrow \infty,
$$


so (3.9) holds and Theorem 3.2 thus applies. The tail behaviour of the lifetime of a parallel system (whose minimal signature is $(2,-1)$ ) with component lifetimes $X_{1}$ and $X_{2}$ is therefore the same as the behaviour of an exponential random variable with rate 1 .

Note that here, when $\theta>0$, we have

$$
\lim _{t \rightarrow \infty} \frac{r_{(1: 2)}(t)}{r_{(1: 1)}(t)}=\lim _{t \rightarrow \infty}(2+2 \theta t)=\infty,
$$

meaning that (3.6) does not hold. This shows that when (2.9) holds, (3.6) is a strictly stronger condition than is (3.9).

Example 3.2. Let $\left(X_{1}, X_{2}, \ldots, X_{n}\right)$ have a Farlie-Gumbel-Morgenstern distribution with standard exponential marginals; that is, suppose that the survival function of $\left(X_{1}, X_{2}, \ldots, X_{n}\right)$ is given by

$$
\bar{F}\left(x_{1}, x_{2}, \ldots, x_{n}\right)=\mathrm{e}^{-\sum_{i=1}^{n} x_{i}}\left(1+\alpha \prod_{i=1}^{n}\left(1-\mathrm{e}^{-x_{i}}\right)\right), \quad\left(x_{1}, x_{2}, \ldots, x_{n}\right) \geq(0,0, \ldots, 0),
$$

where $|\alpha| \leq 1$. It is not hard to verify that if $\alpha \leq 0$ then (2.9) holds (for example, it is not hard to verify that $\bar{F}$ is $\mathrm{RR}_{2}$ in pairs).

For $j<n$, every $j$-dimensional marginal distribution of $\bar{F}$ is the joint distribution of $j$ independent standard exponential random variables. Therefore,

$$
r_{(1: j)}(t)=j, \quad j=1,2, \ldots, n-1 .
$$

A straightforward computation yields

$$
r_{(1: n)}(t)=n-\frac{\alpha n \mathrm{e}^{-t}\left(1-\mathrm{e}^{-t}\right)^{n-1}}{1+\alpha\left(1-\mathrm{e}^{-t}\right)^{n}} .
$$

Note that $\lim _{t \rightarrow \infty} r_{(1: n)}(t)=n$. Therefore,

$$
\lim _{t \rightarrow \infty} \frac{r_{(1: m)}(t)}{r_{(1: i)}(t)}=\frac{m}{i}<\infty, \quad 1 \leq i<m \leq n,
$$

meaning that (3.6) holds. Thus, Theorem 3.1 applies to $\left(X_{1}, X_{2}, \ldots, X_{n}\right)$.

For example, if $r$ is the hazard rate function of, say, System 2 of Table 2, with component lifetimes, $X_{1}, X_{2}, X_{3}$, and $X_{4}$, that have the above survival function (with $n=4$ ), then, by Theorem 3.1, $\lim _{t \rightarrow \infty}\left(r(t) / r_{(1: 3)}(t)\right)=1$. Hence, $\lim _{t \rightarrow \infty} r(t)=3$. Furthermore, if $r$ is the hazard rate function of a $k$-out-of- $n$ system with component lifetimes $X_{1}, X_{2}, \ldots, X_{n}$ as above, then, by Corollary 3.1, $\lim _{t \rightarrow \infty}\left(r(t) / r_{(1: k)}(t)\right)=1$. Hence, $\lim _{t \rightarrow \infty} r(t)=k$.

Note that if $\alpha=0$ then $X_{1}, X_{2}, \ldots, X_{n}$ are independent and identically distributed exponential random variables. Thus, the above limits apply to systems with independent and identically distributed exponential component lifetimes.

Example 3.3. Let $\left(X_{1}, X_{2}, \ldots, X_{n}\right)$ have the Farlie-Gumbel-Morgenstern distribution with gamma(2) marginals; that is, suppose that the survival function of $\left(X_{1}, X_{2}, \ldots, X_{n}\right)$ is given by

$$
\begin{aligned}
\bar{F}\left(x_{1}, x_{2}, \ldots, x_{n}\right)=\left(\prod_{i=1}^{n}\left(1+x_{i}\right)\right) \mathrm{e}^{-\sum_{i=1}^{n} x_{i}}\left(1+\alpha \prod_{i=1}^{n}\left(1-\left(1+x_{i}\right) \mathrm{e}^{-x_{i}}\right)\right) \\
\left(x_{1}, x_{2}, \ldots, x_{n}\right) \geq(0,0, \ldots, 0),
\end{aligned}
$$

where $|\alpha| \leq 1$. It is not hard to verify that if $\alpha \leq 0$ then (2.9) holds. 
For $j<n$, every $j$-dimensional marginal distribution of $\bar{F}$ is the joint distribution of $j$ independent gamma(2) random variables. Therefore,

$$
r_{(1: j)}(t)=\frac{j t}{1+t}, \quad j=1,2, \ldots, n-1 .
$$

A straightforward computation yields

$$
r_{(1: n)}(t)=n-\frac{n}{1+t}-\frac{\alpha n t \mathrm{e}^{-t}\left(1-(1+t) \mathrm{e}^{-t}\right)^{n-1}}{1+\alpha\left(1-(1+t) \mathrm{e}^{-t}\right)^{n}} .
$$

Note that

$$
\lim _{t \rightarrow \infty} r_{(j: n)}(t)=j, \quad j=1,2, \ldots, n
$$

Therefore,

$$
\lim _{t \rightarrow \infty} \frac{r_{(1: m)}(t)}{r_{(1: i)}(t)}=\frac{m}{i}<\infty, \quad 1 \leq i<m \leq n,
$$

meaning that (3.6) holds. Thus, Theorem 3.1 applies to $\left(X_{1}, X_{2}, \ldots, X_{n}\right)$.

Using Theorem 2.1 (rather than Corollary 2.1), Theorem 3.1 can be generalized to the case in which the distribution function of $\left(X_{1}, X_{2}, \ldots, X_{n}\right)$ is not necessarily exchangeable, as follows. Recall from Section 2 that $r_{P}$ denotes the hazard rate function of $\min _{i \in P} X_{i}$.

Theorem 3.3. Let $\left(X_{1}, X_{2}, \ldots, X_{n}\right)$ be an absolutely continuous random vector satisfying (2.2). Let $T=\tau\left(X_{1}, X_{2}, \ldots, X_{n}\right)$, where $\tau$ is a coherent life function with minimal path sets $P_{1}, P_{2}, \ldots, P_{s}$. If

$$
\liminf _{t \rightarrow \infty} \frac{r_{P_{i}}(t)}{r_{P_{1}}(t)}>1, \quad i=2,3, \ldots, s,
$$

and

$$
\limsup _{t \rightarrow \infty} \frac{r_{\{1,2, \ldots, n\}}(t)}{r_{P_{1}}(t)}<\infty
$$

then the hazard rate function, $r$, of $T$ satisfies

$$
\lim _{t \rightarrow \infty} \frac{r(t)}{r_{P_{1}}(t)}=1
$$

Proof. For $A \subseteq\{1,2, \ldots, n\}$, let $P_{A}=\bigcup_{k \in A} P_{k}$. Let $A$ be such that $P_{A} \neq P_{1}$. By (2.3), we have

$$
r_{P_{A}}(t) \geq \max _{k \in A} r_{P_{k}}(t) \quad \text { for all } t \geq 0
$$

Thus, by (3.10),

$$
\liminf _{t \rightarrow \infty} \frac{r_{P_{A}}(t)}{r_{P_{1}}(t)}>1 \quad \text { for all } A \subseteq\{1,2, \ldots, n\}, P_{A} \neq P_{1} .
$$

Furthermore, by (2.3) we also have

$$
r_{\{1,2, \ldots, n\}}(t) \geq r_{P_{A}}(t) \quad \text { for all } A \subseteq\{1,2, \ldots, n\} .
$$

Thus, by (3.11),

$$
\limsup _{t \rightarrow \infty} \frac{r_{P_{A}}(t)}{r_{P_{1}}(t)} \leq \limsup _{t \rightarrow \infty} \frac{r_{\{1,2, \ldots, n\}}(t)}{r_{P_{1}}(t)}<\infty \text { for all } A \subseteq\{1,2, \ldots, n\}, P_{A} \neq P_{1}
$$


Let $\bar{G}$ denote the survival function of $T$. Then, by Equation (3.1) of Block et al. (2003),

$$
\bar{G}(t)=\sum_{A \subseteq\{1,2, \ldots, n\}}(-1)^{|A|+1} \bar{G}_{P_{A}}(t),
$$

where $|A|$ denotes the cardinality of $A$; i.e. $\bar{G}$ is a generalized mixture of the $\bar{G}_{P_{A}}$. The stated result now follows from Lemma 3.3.

It is worth mentioning that if the $\operatorname{limits}_{\lim } \lim _{t \rightarrow \infty}\left(r_{P_{i}}(t) / r_{P_{1}}(t)\right), i=2,3, \ldots, s$, exist, then, under (3.11), the conclusion of Theorem 3.3 holds if (3.10) is replaced by the weaker condition

$$
\liminf _{t \rightarrow \infty} \frac{r_{P_{i}}(t)}{r_{P_{1}}(t)} \geq 1, \quad i=2,3, \ldots, s .
$$

We omit the technical proof of this observation.

It is also worth mentioning that, under (3.10), the conclusion of Theorem 3.3 also holds if (3.11) is replaced by the weaker condition (recall the notation $P_{A}=\bigcup_{k \in A} P_{k}$ )

$$
\lim _{t \rightarrow \infty} \frac{r_{P_{A}}(t) \bar{G}_{P_{A}}(t)}{r_{P_{1}}(t) \bar{G}_{P_{1}}(t)}=0 \text { for all } A \subseteq\{1,2, \ldots, n\}, P_{A} \neq P_{1},
$$

or, equivalently, by

$$
\lim _{t \rightarrow \infty} \frac{g_{P_{A}}(t)}{g_{P_{1}}(t)}=0 \quad \text { for all } A \subseteq\{1,2, \ldots, n\}, P_{A} \neq P_{1},
$$

where $g_{P_{A}}$ and $g_{P_{1}}$ are the density functions of $\min _{i \in P_{A}} X_{i}$ and $\min _{i \in P_{1}} X_{i}$, respectively.

Example 3.4. Let $\left(X_{1}, X_{2}, \ldots, X_{n}\right)$ have a Farlie-Gumbel-Morgenstern distribution with (not necessarily identical) exponential marginals; that is, suppose that the survival function of $\left(X_{1}, X_{2}, \ldots, X_{n}\right)$ is given by

$$
\bar{F}\left(x_{1}, x_{2}, \ldots, x_{n}\right)=\mathrm{e}^{-\sum_{i=1}^{n} \lambda_{i} x_{i}}\left(1+\alpha \prod_{i=1}^{n}\left(1-\mathrm{e}^{-\lambda_{i} x_{i}}\right)\right), \quad\left(x_{1}, x_{2}, \ldots, x_{n}\right) \geq(0,0, \ldots, 0),
$$

where $\lambda_{i}>0, i=1,2, \ldots, n$, and $|\alpha| \leq 1$. It is not hard to verify that if $\alpha \leq 0$ then (2.2) holds.

For $j<n$, every $j$-dimensional marginal distribution of $\bar{F}$ is the joint distribution of $j$ independent exponential random variables. Therefore,

$$
r_{P}(t)=\sum_{j \in P} \lambda_{j} \quad \text { for all } P \subset\{1,2, \ldots, n\}, P \neq\{1,2, \ldots, n\} .
$$

A straightforward computation yields

$$
r_{\{1,2, \ldots, n\}}(t)=\sum_{i=1}^{n} \lambda_{i}-\frac{\alpha \sum_{i=1}^{n}\left(\mathrm{e}^{-\lambda_{i} t} \prod_{j \neq i}\left(1-\mathrm{e}^{-\lambda_{j} t}\right)\right)}{1+\alpha \prod_{i=1}^{n}\left(1-\mathrm{e}^{-\lambda_{i} t}\right)} .
$$

Note that $\lim _{t \rightarrow \infty} r_{\{1,2, \ldots, n\}}(t)=\sum_{i=1}^{n} \lambda_{i}$. Therefore, (3.11) holds. Thus, Theorem 3.3 applies to $\left(X_{1}, X_{2}, \ldots, X_{n}\right)$ in the sense that

$$
\sum_{j \in P_{i}} \lambda_{j}>\sum_{j \in P_{1}} \lambda_{j}, \quad i=2,3, \ldots, s \quad \Longrightarrow \quad \lim _{t \rightarrow \infty} r(t)=\sum_{j \in P_{1}} \lambda_{j}
$$


For example, consider the series-parallel system of Table 1 with component lifetimes, $X_{1}$, $X_{2}$, and $X_{3}$, that have the above survival function (with $n=3$ ). The minimal path sets of this system are $\{1,2\}$ and $\{1,3\}$. Thus, from Theorem 3.3 we obtain

$$
\lim _{t \rightarrow \infty} r(t)=\min \left\{\lambda_{1}+\lambda_{2}, \lambda_{1}+\lambda_{3}\right\}=\lambda_{1}+\min \left(\lambda_{2}, \lambda_{3}\right),
$$

provided that $\lambda_{2} \neq \lambda_{3}$. By letting $\lambda_{2} \rightarrow \lambda_{3}$ (or by using the observation following Theorem 3.3) it can be shown that the above limit holds also when $\lambda_{2}=\lambda_{3}$.

It can similarly be shown, for the parallel-series system of Table 1 with component lifetimes, $X_{1}, X_{2}$, and $X_{3}$, that have the above survival function (with $n=3$ ), that

$$
\lim _{t \rightarrow \infty} r(t)=\min \left\{\lambda_{1}, \lambda_{2}+\lambda_{3}\right\}
$$

If $r$ is the hazard rate function of a $k$-out-of- $n$ system, then the minimal path sets are all the subsets of $\{1,2, \ldots, n\}$ of cardinality $k$. Suppose that the component lifetimes $X_{1}, X_{2}, \ldots, X_{n}$ have the above survival function with $\lambda_{1} \leq \cdots \leq \lambda_{k}<\lambda_{k+1} \leq \cdots \leq \lambda_{n}$. Then, from Theorem 3.3, we obtain

$$
\lim _{t \rightarrow \infty} r(t)=\lambda_{1}+\lambda_{2}+\cdots+\lambda_{k}
$$

Again, by letting $\lambda_{k+1} \rightarrow \lambda_{k}$ (or by using the observation following Theorem 3.3) it can be shown that the above limit holds also when $\lambda_{k+1}=\lambda_{k}$.

\subsection{Some results based on the Samaniego signature}

For exchangeable component lifetimes, Theorems 3.1 and 3.2 describe the tail behaviour of the system hazard rate function in terms of some series system. These theorems are based on the notion of minimal signatures. In this subsection we obtain a similar result based on the Samaniego signature.

Samaniego (1985) defined the signature of a coherent life function $\tau$ with component lifetimes $X_{1}, X_{2}, \ldots, X_{n}$ as the vector, $\boldsymbol{p}=\left(p_{1}, p_{2}, \ldots, p_{n}\right)$, with

$$
p_{i}=\mathrm{P}\left\{\tau\left(X_{1}, X_{2}, \ldots, X_{n}\right)=X_{(i: n)}\right\}, \quad i=1,2, \ldots, n .
$$

Kochar et al. (1999) studied this notion further. It was shown that if $X_{1}, X_{2}, \ldots, X_{n}$ are absolutely continuous, independent, and identically distributed, then

$$
p_{i}=\frac{\text { number of orderings of } X_{1}, \ldots, X_{n} \text { for which the } i \text { th failure causes system failure }}{n !},
$$

In this case the system lifetime survival function, $\bar{F}$, is a mixture of the corresponding order statistic survival functions $\bar{F}_{(i: n)}$ with weights $p_{i}$, as follows:

$$
\bar{F}(t)=\sum_{i=1}^{n} p_{i} \bar{F}_{(i: n)}(t), \quad t \in \mathbb{R} .
$$

The Samaniego signatures of all the systems with three or four components are listed in Shaked and Suarez-Llorens (2003). Navarro et al. (2005) noted that (3.12) and (3.13) continue to hold when the components have an absolutely continuous, exchangeable joint distribution. 
Theorem 3.4. Let $\left(X_{1}, X_{2}, \ldots, X_{n}\right)$ be an absolutely continuous, exchangeable random vector satisfying (2.9). Let $T=\tau\left(X_{1}, X_{2}, \ldots, X_{n}\right)$, where $\tau$ is a coherent life function. Suppose that the Samaniego signature of $\tau$ is of the form $\left(p_{1}, p_{2}, \ldots, p_{j}, 0, \ldots, 0\right)$ for $1 \leq$ $j \leq n$, with $p_{j}>0$. If

$$
\limsup _{t \rightarrow \infty} \frac{r_{(1: n)}(t)}{r_{(1: n-j+1)}(t)}<\infty
$$

then the hazard rate function, $r$, of $T$ satisfies

$$
\lim _{t \rightarrow \infty} \frac{r(t)}{r_{(j: n)}(t)}=1 .
$$

Proof. If (2.9) holds then (2.10) holds. Therefore, for $n-j+1 \leq s \leq n$, we have

$$
\frac{r_{(1: n)}(t)}{r_{(1: s)}(t)} \leq \frac{r_{(1: n)}(t)}{r_{(1: n-j+1)}(t)}, \quad t \in \mathbb{R} .
$$

It follows from (3.14) that

$$
\limsup _{t \rightarrow \infty} \frac{r_{(1: n)}(t)}{r_{(1: s)}(t)}<\infty, \quad n-j+1 \leq s \leq n .
$$

From Corollary 3.1 we thus obtain

$$
\lim _{t \rightarrow \infty} \frac{r_{(n-s+1: n)}(t)}{r_{(1: s)}(t)}=1, \quad n-j+1 \leq s \leq n,
$$

or, equivalently,

$$
\lim _{t \rightarrow \infty} \frac{r_{(m: n)}(t)}{r_{(1: n-m+1)}(t)}=1, \quad 1 \leq m \leq j .
$$

Now write

$$
\frac{r_{(m: n)}(t)}{r_{(m+1: n)}(t)}=\frac{r_{(1: n-m+1)}(t)}{r_{(1: n-m)}(t)} \frac{r_{(m: n)}(t)}{r_{(1: n-m+1)}(t)} \frac{r_{(1: n-m)}(t)}{r_{(m+1: n)}(t)}, \quad t \in \mathbb{R}, 1 \leq m \leq j-1 .
$$

By letting $t \rightarrow \infty$ in (3.17) and using (3.16), we see that

$$
\liminf _{t \rightarrow \infty} \frac{r_{(m: n)}(t)}{r_{(m+1: n)}(t)}=\liminf _{t \rightarrow \infty} \frac{r_{(1: n-m+1)}(t)}{r_{(1: n-m)}(t)}, \quad 1 \leq m \leq j-1 .
$$

From (3.7) (which, by (2.9) and (2.10), holds for all $j$ and $i$ with $1 \leq j<i \leq n$, using the notation of (3.7)), we see that $\liminf _{t \rightarrow \infty}\left(r_{(1: n-m+1)}(t) / r_{(1: n-m)}(t)\right)>1$. Hence,

$$
\begin{array}{r}
\liminf _{t \rightarrow \infty} \frac{r_{(m: n)}(t)}{r_{(j: n)}(t)} \geq \liminf _{t \rightarrow \infty} \frac{r_{(m: n)}(t)}{r_{(m+1: n)}(t)} \liminf _{t \rightarrow \infty} \frac{r_{(m+1: n)}(t)}{r_{(m+2: n)}(t)} \cdots \liminf _{t \rightarrow \infty} \frac{r_{(j-1: n)}(t)}{r_{(j: n)}(t)}>1, \\
1 \leq m \leq j-1 .
\end{array}
$$

Again by letting $t \rightarrow \infty$ in (3.17) and using (3.16), we see that

$$
\limsup _{t \rightarrow \infty} \frac{r_{(m: n)}(t)}{r_{(m+1: n)}(t)}=\limsup _{t \rightarrow \infty} \frac{r_{(1: n-m+1)}(t)}{r_{(1: n-m)}(t)}, \quad 1 \leq m \leq j-1 .
$$


Also, by (2.10) and (3.15),

$$
\limsup _{t \rightarrow \infty} \frac{r_{(1: n-m+1)}(t)}{r_{(1: n-m)}(t)} \leq \limsup _{t \rightarrow \infty} \frac{r_{(1: n)}(t)}{r_{(1: n-m)}(t)}<\infty, \quad 1 \leq m \leq j-1 .
$$

Therefore,

$$
\limsup _{t \rightarrow \infty} \frac{r_{(m: n)}(t)}{r_{(m+1: n)}(t)}<\infty, \quad 1 \leq m \leq j-1 .
$$

Hence,

$$
\begin{gathered}
\limsup _{t \rightarrow \infty} \frac{r_{(m: n)}(t)}{r_{(j: n)}(t)} \leq \limsup _{t \rightarrow \infty} \frac{r_{(m: n)}(t)}{r_{(m+1: n)}(t)} \limsup _{t \rightarrow \infty} \frac{r_{(m+1: n)}(t)}{r_{(m+2: n)}(t)} \cdots \limsup _{t \rightarrow \infty} \frac{r_{(j-1: n)}(t)}{r_{(j: n)}(t)}<\infty \\
1 \leq m \leq j-1
\end{gathered}
$$

We now apply Lemma 3.3 to the mixture (3.13), with (3.18) and (3.19) playing the roles of (3.3) and (3.4) in that lemma. This yields the stated result.

An interesting relationship between the Samaniego signature and the minimal signature follows from Theorems 3.1 and 3.4, as described in the next result.

Theorem 3.5. Suppose that the Samaniego signature of a coherent system is of the form $\left(p_{1}, p_{2}, \ldots, p_{j}, 0,0, \ldots, 0\right)$ for $1 \leq j \leq n$, with $p_{j}>0$, and that the minimal signature of the same system is of the form $\left(0,0, \ldots, 0, a_{i}, a_{i+1}, \ldots, a_{n}\right)$ for $1 \leq i \leq n$, with $a_{i}>0$. Then $i+j=n+1$.

Proof. Example 3.2 shows that there exists an absolutely continuous, exchangeable random vector such that (2.9) holds, and also such that (3.6) holds for all $i$ and $m$ with $1 \leq i \leq m \leq n$. It follows that, for this random vector, (3.8) holds for all $k, 1 \leq k \leq n$, and (3.14) holds for all $j, 1 \leq j \leq n$. Moreover, in this example $\lim _{t \rightarrow \infty} r_{(1: i)}(t)=i, i=1,2, \ldots, n$. Hence, a combination of the conclusions of Theorem 3.1, Corollary 3.1, and Theorem 3.4 yields the stated result.

\section{References}

Baggs, G. E. ANd Nagaraja, H. N. (1996). Reliability properties of order statistics from bivariate exponential distributions. Commun. Statist. Stoch. Models 12, 611-631.

Barlow, R. E. and Proschan, F. (1975). Statistical Theory of Reliability and Life Testing. Probability Models. Holt, Rinehart and Winston, New York.

Block, H. And Joe, H. (1997). Tail behavior of the failure rate functions of mixtures. Lifetime Data Anal. 3, 269-288.

Block, H. W., LI, Y. AND SAVITs, T. H. (2003). Initial and final behaviour of failure rate functions for mixtures and systems. J. Appl. Prob. 40, 721-740.

Block, H. W., Savits, T. H. and Shaked, M. (1982). Some concepts of negative dependence. Ann. Prob. 10, 765-772.

Freund, J. E. (1961). A bivariate extension of the exponential distribution. J. Amer. Statist. Assoc. 56, 971-977.

Holt, J. D. (1978). Competing risk analyses with special reference to matched pair experiments. Biometrika 65, $159-165$.

Johnson, N. L. And Kotz, S. (1975). A vector multivariate hazard rate. J. Multivariate Anal. 5, 53-66, 498.

KARLin, S. AND RinOtT, Y. (1980). Classes of orderings of measures and related correlation inequalities. II. Multivariate reverse rule distributions. J. Multivariate Anal. 10, 499-516.

Kochar, S., Mukerjee, H. and Samaniego, F. J. (1999). The "signature" of a coherent system and its application to comparisons among systems. Naval Res. Logistics 46, 507-523.

Korwar, R. (2003). On stochastic orders for the lifetime of a $k$-out-of-n system. Prob. Eng. Inf. Sci. 17, $137-142$.

LI, Y. (2005). Asymptotic baseline of the hazard rate function of mixtures. J. Appl. Prob. 42, 892-901.

Marshall, A. W. (1975). Some comments on the hazard gradient. Stoch. Process. Appl. 3, 293-300. 
NaVARro, J. AND HeRnAndez, P. J. (2004a). How to obtain bathtub-shaped failure rate models from normal mixtures. Prob. Eng. Inf. Sci. 18, 511-531.

Navarro, J. and Hernandez, P. J. (2004b). Properties of the failure rate functions of generalized mixtures. Tech. Rep., Facultad de Matematicas, Universidad de Murcia.

Navarro, J., Ruiz, J. M. and Sandoval, C. J. (2004). Properties of coherent systems with dependent components. To appear in Commun. Statist. Theory Meth.

Navarro, J., Ruiz, J. M. And Sandoval, C. J. (2005). A note on comparisons among coherent systems with dependent components using signatures. Statist. Prob. Lett. 72, 179-185.

Prentice, R. L. et al. (1978). The analysis of failure times in the presence of competing risks. Biometrics 34, 541-554.

Samaniego, F. J. (1985). On the closure of the IFR class under formation of coherent systems. IEEE Trans. Reliab. 34, 69-72.

Scarsini, M. And Shaked, M. (1999). Distributions with known initial hazard rate functions. J. Statist. Planning Infer. 78, 39-55.

Shaked, M. And Shanthikumar, J. G. (1994). Stochastic Orders and Their Applications. Academic Press, Boston, MA.

Shaked, M. And Suarez-Llorens, A. (2003). On the comparison of reliability experiments based on the convolution order. J. Amer. Statist. Assoc. 98, 693-702. 